\title{
Hemodilution and surgical hemostasis contribute significantly to transfusion requirements in patients undergoing coronary artery bypass
}

Sandra Dial, MD, MSc, Eugene Delabays, MD, Martin Albert, MD, Anne Gonzalez, MD, Jordan Camarda, BSc, Adora Law, BSc, and Dick Menzies, MD, MSc

Extra illustrations are available online. $\beta$
From McGill University, Montreal, Quebec, Canada.

Supported by the MUHC Research Institute and FRSQ.

Received for publication Nov 3, 2004; revisions received Feb 8, 2005; accepted for publication Feb 15, 2005

Address for reprints: Sandra Dial, MD, MSc, Department of Critical Care, SMBD Jewish General Hospital, McGill University, Montreal, and Respiratory Epidemiology and Clinical Research Unit, Montreal Chest Institute, 3650 St Urbain, Room K1.14, Montreal, Quebec, Canada, H2X 2P4 (E-mail: sandra.dial@mcgill.ca).

J Thorac Cardiovasc Surg 2005;130:654-61

$0022-5223 / \$ 30.00$

Copyright $(92005$ by The American Association for Thoracic Surgery

doi:10.1016/j.jtcvs.2005.02.025
Objective: We sought to determine the incidence of and risk factors for the development of low intraoperative hematocrit levels and of excessive postoperative bleeding in patients undergoing coronary artery bypass grafting, whether the risk factors are the same, and their effect on blood product transfusions.

Methods: We performed a prospective cohort study of 613 adult patients who underwent coronary artery bypass grafting in 3 tertiary, university-affiliated hospitals during the period from October 1, 2000, to March 31, 2001.

Results: Low intraoperative hematocrit levels $(<19 \%)$ were found in $131(24 \%)$ patients who had operations performed with extracorporeal circulation compared with in $3(4 \%)$ patients with operations performed off pump. In multivariate analysis this was associated with older age, female sex, lower preoperative hemoglobin levels, lower body surface area, longer duration on bypass, and use of higher total volumes with more hydroxyethyl starch in the circuit. Low intraoperative hematocrit levels did not predict excessive postoperative hemorrhage $(>1 \mathrm{~L}$ of mediastinal drainage in the first 12 hours). This occurred in 26\% ( $\mathrm{n}=140)$ of patients undergoing on-pump operations and in 25\% of patients undergoing off-pump operations and in multivariate analysis was associated with male sex, longer pump times, not receiving aprotinin, and operations performed by certain surgeons but not with total circuit or hydroxyethyl starch volume.

Conclusions: We observed that the risk factors for the development of a low intraoperative hematocrit level and excessive postoperative bleeding differed. Our results suggest that decreasing these outcomes in patients undergoing cardiac surgery requires a comprehensive approach, including limiting hemodilution, particularly in female subjects with lower preoperative hemoglobin levels, and careful attention to surgical hemostasis.

$\mathrm{P}$ atients who undergo cardiac surgery commonly receive blood product transfusions, but there are substantial and unexplained differences in rates in different institutions. ${ }^{1,2}$ Consensus recommendations are to consider intraoperative transfusion of packed cells if the hematocrit level decreases to less than $19 \%$ intraoperatively. ${ }^{3,4}$ Drainage in excess of $1 \mathrm{~L}$ postoperatively is associated with accelerated transfusion of blood products in the order of greater than 5 units. ${ }^{2,5}$ Both excessive bleeding and low hematocrit levels are common and associated with worse outcomes, ${ }^{6-8}$ yet these have not been well characterized. ${ }^{2,6}$ Because of the assumption that risk factors for perioperative blood product transfusions ${ }^{3,9,10}$ and for bleeding are the same, preventive strategies have focused on agents promoting or limiting preoperative use of agents that impair hemostasis. ${ }^{11-14}$ However, intraoperative anemia might be related to hemodilution, but this has received scant attention. ${ }^{15-17}$ It is also unclear whether the substantial differences in blood product 
use related to cardiac surgery ${ }^{1}$ stem from different transfusion practices or protocols, intraoperative antifibrinolytic use, surgical technique, or differences in patient populations.

After observing differences in practices in cardiac surgery in the 3 affiliated hospitals at our university, we prospectively evaluated the occurrence and risk factors for excessive postoperative hemorrhage and low intraoperative hematocrit values.

\section{Methods}

\section{Setting and Study Population}

The study was conducted in 3 general, tertiary, university-affiliated adult hospitals. In hospital A approximately 800 cardiac surgery procedures are performed annually by 4 surgeons, whereas in hospitals B and C approximately 500 such procedures are performed annually, primarily by 2 surgeons at each site; one surgeon operated in both hospitals B and C. In hospital A, a total volume of $2000 \mathrm{~mL}$ was used initially in the extracorporeal circuit in all patients, $750 \mathrm{~mL}$ of which was hydroxyethyl starch (HES), whereas in hospitals B and C, the total volume was $1400 \mathrm{~mL}$, of which $500 \mathrm{~mL}$ was HES.

None of the hospitals had written transfusion protocols. As antifibrinolytic agents, aprotinin was routinely used in hospital A, whereas tranexamic acid was routinely used in the other 2 hospitals. During the time of the study, aprotinin was temporarily unavailable from the manufacturer, and as a result, 46 patients in hospital A underwent cardiac surgery without any antifibrinolytic agent. All patients were admitted to the intensive care unit (ICU) postoperatively, and retransfusion of shed mediastinal blood was practiced at all sites.

Data were collected prospectively on 613 consecutive patients who had undergone coronary artery bypass graft $(\mathrm{CABG})$ procedures only without any valvular operations from October 1, 2000, to March 31, 2001, in hospitals B and C and from December 1, 2000 to March 31, 2001, in hospital A. A total of 545 procedures were performed on pump, and 68 were performed off pump across the 3 sites. The study was approved by the research ethics committees of the participating institutions.

\section{Data Collection}

The following data were collected from standardized data collection sheets.

Preoperative characteristics included age, sex, body surface area, Parsonnet score ${ }^{18}$ history of prior cardiac surgery, premorbid diagnoses (eg, diabetes mellitus, hypertension, renal failure, coagulation disorders, and left ventricular dysfunction), and whether surgical intervention was required within 6 hours (ie, an emergency procedure). We also recorded preoperative use of drugs that impair hemostasis, such as aspirin, clopidogrel, heparin, or warfarin, as well as preoperative hemoglobin level, international normalized ratio, partial thromboplastin time, and platelet count.

Intraoperative factors included identity of the primary surgeon, antifibrinolytic agent used, time on extracorporeal circulation, lowest intraoperative hematocrit level recorded, type and number of blood products transfused intraoperatively, highest recorded acti- vated clotting time, and total number of grafts, including whether arterial or vein grafts were used.

Postoperative factors included hemoglobin and coagulation parameters, need for exploratory reoperation, mortality, length of stay in the ICU and length of hospitalization, mediastinal drainage in the first 12 hours, and number and type of blood products transfused in the postoperative period.

\section{Data Analysis}

Two outcomes were selected for analysis: (1) severe intraoperative anemia, which was defined as the development of an intraoperative hematocrit level of less than $19 \%$, and (2) excessive postoperative bleeding, which was defined as mediastinal drainage of greater than $1000 \mathrm{~mL}$ in the first 12 hours postoperatively. Blood product transfusions and re-exploration have been suggested as surrogates for bleeding, ${ }^{9}$ but there is no consensus regarding the criteria for transfusion $^{2}$ or reoperation. ${ }^{19,20}$ These outcomes were also chosen as potential outcomes that would be important triggers for transfusion that would decrease, although not completely control, the effect of variability in practice.

The postoperative period was defined as the time in the ICU up to a maximum of 3 days for patients who stayed longer in the ICU. The perioperative period was defined as the intraoperative and postoperative periods combined. Patients having their procedure performed off pump were analyzed separately.

Differences between patients who did or did not have the 2 major outcomes were tested for statistical significance by using the Student $t$ test for normally distributed data and the Wilcoxon rank test for nonnormal continuous data, whereas $\chi^{2}$ tests were used for categorical variables. Multivariate logistic regression was performed to obtain adjusted estimates of the odds with $95 \%$ confidence intervals of patient, procedure, and provider factors associated with the development of intraoperative anemia and excessive postoperative bleeding. Variables were included in the multivariate models if they were significantly associated or if there was evidence of a substantial effect on univariate analysis, as well as clinical factors associated with transfusions in previous studies. A nested model was used (PROC GENMOD-SAS) in which the surgeons using the same intraoperative initial circuit volume and similar type of antifibrinolytic agent were compared with each other to assess the independent effect of surgeon. Statistical testing was performed with SAS 8.2 (SAS Institute, Cary, NC).

\section{Results}

The preoperative variables of the 545 patients undergoing CABG with extracorporeal circulation were similar among the 3 hospitals (Table 1), and data from all 3 hospitals were subsequently combined. The proportion of patients receiving aspirin and heparin preoperatively was significantly different at the 3 sites, with corresponding differences in the preoperative coagulation profile. Intraoperative pump time and the number of grafts were also different. In hospital A, which used larger circuit and HES volumes, significantly more patients had severe intraoperative anemia, but fewer had excessive postoperative bleeding. Hospital $\mathrm{C}$ had the lowest re-exploration rate, even though it had the highest proportion of patients with excessive postoperative bleeding. 
TABLE 1. Preoperative, intraoperative, and postoperative characteristics and outcomes of all patients in the 3 hospitals

\begin{tabular}{|c|c|c|c|c|}
\hline & Hospital A & Hospital B & Hospital C & $P$ value \\
\hline No. of patients & 214 & 189 & 142 & \\
\hline Age, y (mean [SD]) & $66.4(10.2)$ & $66.3(10.2)$ & $65.2(9.6)$ & NS \\
\hline Female sex, $\mathrm{n}(\%)$ & $48(22)$ & $46(24)$ & $38(27)$ & NS \\
\hline Body surface area (mean [SD]) & $1.88(0.22)$ & $1.90(0.21)$ & $1.88(0.28)$ & NS \\
\hline Parsonnet score (mean [SD]) & $10.7(9.5)$ & $10.2(8.6)$ & $9.5(6.9)$ & NS \\
\hline Patient with score $>20, \mathrm{n}(\%)$ & $30(14)$ & $30(16)$ & $11(8)$ & .04 \\
\hline \multicolumn{5}{|l|}{ Medical history } \\
\hline Previous cardiac surgery, $\mathrm{n}(\%)$ & $8(3.8)$ & $5(2.6)$ & $7(5)$ & NS \\
\hline \multicolumn{5}{|l|}{ Preop hematology } \\
\hline Hemoglobin (mean [SD]) & $133.5(15.9)$ & $133.1(15.6)$ & $134.6(18.5)$ & NS \\
\hline Platelet count (mean [SD]) & $246(66)$ & $225(73)$ & $246(73)$ & .003 \\
\hline INR (mean [SD]) & $1.07(0.10)$ & $1.14(0.31)$ & $1.07(0.16)$ & $<.001$ \\
\hline PTT (mean [SD]) & $45(27)$ & $54(39)$ & $41(29)$ & .001 \\
\hline \multicolumn{5}{|l|}{ Preop medications } \\
\hline Heparin (full dose), n (\%) & $49(23)$ & $109(58)$ & $68(48)$ & .02 \\
\hline Aspirin, n (\%) & $172(81)$ & $92(49)$ & $104(73)$ & .001 \\
\hline \multicolumn{5}{|l|}{ Intraop variables } \\
\hline No. of grafts (mean [SD]) & $3.1(0.9)$ & $3.6(1.1)$ & $3.8(1.1)$ & .001 \\
\hline Bypass time, min (mean [SD]) & $77(27)$ & $87(32)$ & $90(35)$ & .001 \\
\hline Tranexamic acid, n (\%) & $8(4)$ & $174(92)$ & $140(99)$ & .001 \\
\hline Aprotinin, n (\%) & $161(76)$ & $6(3)$ & 0 & \\
\hline No antifibrinolytic agent, n (\%) & 44 & $9(5)$ & $2(1)$ & \\
\hline \multicolumn{5}{|l|}{ Pump volume added initially } \\
\hline Total & 2000 & 1400 & 1400 & \\
\hline Colloid & 750 & 500 & 500 & \\
\hline \multicolumn{5}{|l|}{ Postop variables } \\
\hline Platelet count (mean [SD]) & $148(60)$ & $147(62)$ & $142(54)$ & NS \\
\hline INR (mean [SD]) & $1.60(0.24)$ & $1.36(0.24)$ & $1.28(0.28)$ & $<.001$ \\
\hline PTT (mean [SD]) & $45(13)$ & $40(23)$ & $56(41)$ & $<.001$ \\
\hline \multicolumn{5}{|l|}{ Outcomes } \\
\hline \multicolumn{5}{|l|}{ Intraop НCT } \\
\hline Lowest (mean [SD]) & $20.6(4.4)$ & $22.4(3.5)$ & $21.4(4.0)$ & $<.0001$ \\
\hline Patients with HCT $<19 \%$, n (\%) & $65(30)$ & $32(17)$ & $34(24)$ & .006 \\
\hline \multicolumn{5}{|l|}{ Postop drainage in the first $12 \mathrm{~h}$} \\
\hline Mean (SD) & $765(615)$ & $800(555)$ & $965(730)$ & .01 \\
\hline Patients bleeding $>1 \mathrm{~L}, \mathrm{n}(\%)$ & $42(20)$ & $47(25)$ & $50(35)$ & .005 \\
\hline Patients transfused, $\mathrm{n}(\%)$ & $110(51)$ & $89(46)$ & $83(51)$ & NS \\
\hline Hemoglobin at ICU discharge (mean [SD]) & $95(13)$ & $100(14)$ & $100(11)$ & $<.001$ \\
\hline \multicolumn{5}{|l|}{ Length of stay, $d$} \\
\hline In ICU (mean [median]) & $2.2(1)$ & $3.2(1)$ & $2.2(1)$ & NS \\
\hline Total in hospital (mean [median]) & $9.5(7)$ & $11.7(7)$ & $10(7)$ & NS \\
\hline Re-exploratory surgery, $\mathrm{n}(\%)$ & $8(3.7)$ & $14(7.4)$ & $2(1.4)$ & .008 \\
\hline Died, $\mathrm{n}(\%)$ & $4(1.9)$ & $7(3.7)$ & $6(4.2)$ & NS \\
\hline
\end{tabular}

$S D$, Standard deviation; $N S$, not significant; INR, international normalized ratio; $P T T$, partial thromboplastin time; $H C T$, hematocrit; ICU, intensive care unit.

In total, $131(24 \%)$ of the 545 patients whose procedures were performed on pump had severe intraoperative anemia. In univariate analysis this was significantly more likely in older female patients and patients with a lower body surface area and lower preoperative hemoglobin levels. Intraoperative anemia was also associated with higher Parsonnet scores or receipt of larger total volume and larger volumes of colloid in the extracorporeal circuit (Table 2) but not with use of aprotinin or tranexamic acid. The occurrence of intraoperative anemia by surgeon varied from $18 \%$ to $49 \%$ (Figure E1). Patients who had intraoperative anemia were more likely to be transfused packed cells (Figure E2), had lower ICU discharge hemoglobin levels, longer hospital and ICU lengths of stay, and higher mortality; however, they did not have an increased frequency of excessive postoperative bleeding. 
TABLE 2. Comparison of characteristics and outcomes between patients who did and did not have the lowest intraoperative hematocrit level of less than $19 \%$ in patients whose procedures were performed on pump

\begin{tabular}{|c|c|c|c|}
\hline & $\begin{array}{c}\text { Lowest intraop } \\
\text { HCT } \geq 19 \%\end{array}$ & $\begin{array}{c}\text { Lowest intraop } \\
\text { HCT }<19 \%\end{array}$ & $\begin{array}{c}\text { RR }(95 \% \mathrm{CI}) \\
\text { or } \boldsymbol{P} \text { value }\end{array}$ \\
\hline No. of patients & 414 & 131 & \\
\hline Age, y (mean [SD]) & $65(10)$ & $69.7(9.6)$ & $<.001$ \\
\hline Female sex, $\mathrm{n}(\%)$ & $61(15)$ & $71(31)$ & $3.7(2.8-4.9)$ \\
\hline Body surface area (mean [SD]) & $1.93(0.22)$ & $1.75(0.2)$ & $<.001$ \\
\hline Parsonnet score (mean [SD]) & $9.3(7.5)$ & $13.2(10.8)$ & $<.001$ \\
\hline Patient with score $>20, \mathrm{n}(\%)$ & $41(10)$ & $31(24)$ & $2.0(1.5-2.8)$ \\
\hline \multicolumn{4}{|l|}{ Medical history } \\
\hline Previous cardiac surgery, n (\%) & $12(3)$ & $8(6)$ & $2.1(0.9-5.0)$ \\
\hline LVEF $<50 \%, \mathrm{n}(\%)$ & $117(28)$ & $36(27)$ & $1.0(0.7-1.3)$ \\
\hline \multicolumn{4}{|l|}{ Preop hematology } \\
\hline Hemoglobin (mean [SD]) & $137.2(15.4)$ & $122.5(15.1)$ & $<.001$ \\
\hline Platelet count (mean [SD]) & $235(73)$ & $250(62)$ & .01 \\
\hline INR (mean [SD]) & $1.1(0.7)$ & $1.1(0.4)$ & NS \\
\hline PTT (mean [SD]) & $47(32)$ & $47(33)$ & NS \\
\hline \multicolumn{4}{|l|}{ Preop medications } \\
\hline Heparin (full dose), n (\%) & $175(42)$ & $51(39)$ & $0.9(0.7-1.2)$ \\
\hline Aspirin, n (\%) & $275(66)$ & $93(71)$ & $1.2(0.8-1.6)$ \\
\hline \multicolumn{4}{|l|}{ Intraop variables } \\
\hline No. of grafts (mean [SD]) & $3.5(1.1)$ & $3.4(1.0)$ & NS \\
\hline Bypass time, min (mean [SD]) & $82(30)$ & $89(36)$ & NS \\
\hline Tranexamic acid, $\mathrm{n}(\%)$ & $253(61)$ & $69(53)$ & $0.8(0.6-1.0)$ \\
\hline Aprotinin, $\mathrm{n}(\%)$ & $123(30)$ & $44(33)$ & $1.1(0.8-1.6)$ \\
\hline No antifibrinolytic agent, n (\%) & $38(9)$ & $18(14)$ & $1.4(0.9-2.1)$ \\
\hline \multicolumn{4}{|l|}{ Pump volume added initially } \\
\hline Total (mean [SD]) & $1614(288)$ & $1697(301)$ & .004 \\
\hline Colloid (mean [SD]) & $383(463)$ & $522(477)$ & .003 \\
\hline \multicolumn{4}{|l|}{ Outcomes } \\
\hline \multicolumn{4}{|l|}{ Intraop transfusions of packed cells } \\
\hline No. of units transfused (mean [SD]) & $0.6(1.4)$ & $2.2(2.6)$ & $<.001$ \\
\hline Patients transfused, n (\%) & $109(26)$ & $98(75)$ & $2.8(2.1-3.8)$ \\
\hline \multicolumn{4}{|l|}{ Postop drainage in first $12 \mathrm{~h}$} \\
\hline Mean [SD] & $818(629)$ & $865(640)$ & NS \\
\hline \multicolumn{4}{|l|}{ Patients with $>1 \mathrm{~L}$ drainage } \\
\hline Postop transfusions of packed cells, $\mathrm{n}(\%)$ & $99(24)$ & $41(31)$ & $1.3(1.0-1.8)$ \\
\hline No. of units transfused (mean [SD]) & $0.7(1.8)$ & $1.8(3.0)$ & .0001 \\
\hline Patients transfused, n (\%) & $97(23)$ & $74(56)$ & $2.8(2.1-3.8)$ \\
\hline \multicolumn{4}{|l|}{ Total intraop and ICU postoperative transfusions } \\
\hline No. units transfused (mean [SD]) & $1.4(2.6)$ & $4.0(4.3)$ & $<.0001$ \\
\hline Patients transfused, $\mathrm{n}(\%)$ & $166(40)$ & $116(88)$ & $7.2(4.3-12)$ \\
\hline ICU discharge hemoglobin & $99(13)$ & $93(13)$ & $<.0001$ \\
\hline \multicolumn{4}{|l|}{ Length of stay, $d$} \\
\hline ICU (mean [median]) & $2.4(1)$ & $3.4(2)$ & .007 \\
\hline Total in hospital (mean [median]) & $9.9(7)$ & $12.1(8)$ & .003 \\
\hline Re-exploratory surgery, n (\%) & $16(3.9)$ & $8(6.1)$ & $1.6(0.7-3.6)$ \\
\hline Died, $\mathrm{n}(\%)$ & $9(2.2)$ & $8(6.1)$ & $2.8(1.1-7.1)$ \\
\hline
\end{tabular}

$H C T$, Hematocrit; $R R$, risk ratio; $C l$, confidence interval; $S D$, standard deviation; $L V E F$, left ventricular ejection fraction; INR, international normalized ratio; $P T T$, partial thromboplastin time; ICU, intensive care unit.

Of the 68 other patients who had their procedures performed off pump and therefore would not have received any priming volumes, only $3(4 \%)$ had intraoperative anemia (data not shown).
Excessive postoperative hemorrhage occurred in $26 \%$ of the patients whose procedures were performed on pump. The only preoperative patient characteristic associated with excessive postoperative bleeding in univariate analysis was 
TABLE 3. Comparison of characteristics and outcomes between patients who had less than or more than $1 \mathrm{~L}$ of mediastinal drainage in the first 12 hours postoperatively in patients whose procedures were performed on pump

\begin{tabular}{|c|c|c|c|}
\hline & $\begin{array}{c}\text { Drainage } \\
<1 \mathrm{~L}\end{array}$ & $\begin{array}{c}\text { Drainage } \\
>1 \mathrm{~L}\end{array}$ & $\begin{array}{c}\text { RR (95\% CI) or } \\
\quad P \text { value }\end{array}$ \\
\hline No. of patients & 405 & 140 & \\
\hline Age, y (mean [SD]) & $66.4(10)$ & $65.0(10)$ & NS \\
\hline Female sex, $\mathrm{n}(\%)$ & $112(28)$ & $20(14)$ & $0.5(0.3-0.8)$ \\
\hline Body surface area (mean [SD]) & $1.89(0.22)$ & $1.89(0.26)$ & NS \\
\hline Parsonnet score (mean [SD]) & $10.4(8.5)$ & $9.7(8.8)$ & NS \\
\hline Patients with score $>20, \mathrm{n}(\%)$ & $52(13)$ & $19(14)$ & $1.1(0.6-1.7)$ \\
\hline \multicolumn{4}{|l|}{ Medical history } \\
\hline Previous cardiac surgery, n (\%) & $15(3.7)$ & $5(3.5)$ & $1.0(0.4-2.6)$ \\
\hline LVEF $<50 \%, \mathrm{n}(\%)$ & $109(27)$ & $44(31)$ & $1.2(0.9-1.6)$ \\
\hline \multicolumn{4}{|l|}{ Preop hematology } \\
\hline Hemoglobin (mean [SD]) & $132(16.2)$ & $136(17)$ & .05 \\
\hline Platelet count (mean [SD]) & $236(68)$ & $245(77)$ & NS \\
\hline INR (mean [SD]) & $1.1(0.1)$ & $1.1(0.4)$ & NS \\
\hline PTT (mean [SD]) & $47(30)$ & 47 (37) & NS \\
\hline \multicolumn{4}{|l|}{ Preop medications } \\
\hline Heparin (full dose), n (\%) & $174(43)$ & $52(37)$ & $0.8(0.6-1.1)$ \\
\hline Aspirin, n (\%) & $272(67)$ & $96(68)$ & $1.1(0.8-1.4)$ \\
\hline \multicolumn{4}{|l|}{ Intraop variables } \\
\hline No. of grafts (mean [SD]) & $3.4(1.1)$ & $3.7(1.0)$ & .007 \\
\hline Bypass time, min (mean [SD]) & $81(2.7)$ & $92(39)$ & .001 \\
\hline Tranexamic acid, n (\%) & $229(56)$ & $92(66)$ & $1.4(1.0-1.9)$ \\
\hline Aprotinin, $\mathrm{n}(\%)$ & $141(35)$ & $26(19)$ & $0.5(0.4-0.8)$ \\
\hline No antifibrinolytic agent, n (\%) & $35(9)$ & $22(15)$ & $1.6(1.1-2.3)$ \\
\hline Lowest intraop HCT (mean [SD]) & $21.5(4.0)$ & $21.2(4.1)$ & NS \\
\hline Patients with intraop HCT <19\%, n (\%) & $90(22)$ & $41(29)$ & $1.3(1.0-1.8)$ \\
\hline Transfused any packed cells in OR, $\mathrm{n}(\%)$ & $139(34)$ & $68(48)$ & $1.5(1.2-2.0)$ \\
\hline Mean no. of units transfused (mean [SD]) & $0.7(1.2)$ & $1.7(2.9)$ & $<.0001$ \\
\hline \multicolumn{4}{|l|}{ Postop variables } \\
\hline Platelet count (mean [SD]) & $147(58)$ & $144(63)$ & NS \\
\hline INR (mean [SD]) & $1.1(0.3)$ & $1.1(0.1)$ & NS \\
\hline PTT (mean [SD]) & $47(33)$ & $47(29)$ & NS \\
\hline \multicolumn{4}{|l|}{ Outcomes } \\
\hline \multicolumn{4}{|l|}{ Postop transfusions of packed cells } \\
\hline No. of patients transfused, $\mathrm{n}(\%)$ & $91(22)$ & $80(57)$ & $2.9(2.2-3.9)$ \\
\hline No. of units transfused (mean [median]) & $0.5(0)$ & $2.5(1)$ & $<.0001$ \\
\hline \multicolumn{4}{|l|}{ Total intraop and ICU postop transfusions } \\
\hline No. of units transfused (mean [SD]) & $1.2(1.9)$ & $4.2(5.1)$ & $<.0001$ \\
\hline Patients transfused, $\mathrm{n}(\%)$ & $181(45)$ & $101(72)$ & $3.0(2.1-4.4)$ \\
\hline ICU discharge hemoglobin (mean [SD]) & $99(13)$ & $93(12)$ & $<.001$ \\
\hline \multicolumn{4}{|l|}{ Length of stay, $d$} \\
\hline ICU (mean [median]) & $2.2(1)$ & $3.7(2)$ & $<.001$ \\
\hline Total in hospital (mean [median]) & $10.1(7)$ & $11.1(7)$ & NS \\
\hline Re-exploratory surgery, n (\%) & $4(1)$ & $20(14)$ & $14.5(5.0-42)$ \\
\hline Died, $\mathrm{n}(\%)$ & $6(1.5)$ & $11(8)$ & $5.3(2.0-14.1)$ \\
\hline
\end{tabular}

$R R$, Risk ratio; $C l$, confidence interval; $S D$, standard deviation; $L V E F$, left ventricular ejection fraction; INR, international normalized ratio; $P T T$, partial thromboplastin time; $H C T$, hematocrit; $O R$, operating room; ICU, intensive care unit.

male sex (Table 3). Excessive postoperative bleeding was more likely with longer bypass time, higher number of grafts performed, and certain surgeons (Figure E3) and less likely if aprotinin or tranexamic acid was used. Bleeding was not associated with preoperative aspirin or heparin or with coagula- tion parameters measured immediately postoperatively. Patients with excessive postoperative bleeding were more likely to be transfused packed cells (Figure E4), had longer ICU lengths of stay, were more likely to undergo re-exploratory surgery, and were more likely to die. 
TABLE 4. Multivariate analysis of factors associated with the development of intraoperative anemia and excessive postoperative bleeding in patients whose procedures were performed on pump*

\begin{tabular}{|c|c|c|}
\hline Characteristics & $\begin{array}{l}\text { Lowest intraop HCT }<19 \% \text {, } \\
\text { adjusted odds ratio }(95 \% \mathrm{CI})\end{array}$ & $\begin{array}{l}\text { Mediastinal drainage }>1 \mathrm{~L} \text { in the first } \\
12 \text { postop hours, odds ratio ( } 95 \% \mathrm{CI})\end{array}$ \\
\hline Older age (increase of $5[\mathrm{eg}, 65-70])$ & $1.1(0.9-1.2)$ & $0.9(0.8-1.0)$ \\
\hline Female sex & $3.7(2.1-6.5)$ & $0.4(0.2-0.7)$ \\
\hline Lower preoperative hemoglobin (decrease of 10) & $1.6(1.4-1.9)$ & $0.9(0.8-1.05)$ \\
\hline Lower body surface area (decrease of 0.1 ) & $1.2(1.1-1.4)$ & $0.9(0.8-1.0)$ \\
\hline Longer bypass time (increase of $30 \mathrm{~min}$ ) & $1.4(1.1-1.8)$ & $1.6(1.3-2.0)$ \\
\hline Heparin & $0.7(0.4-1.2)$ & $0.8(0.5-1.3)$ \\
\hline Aspirin & $1.3(0.7-2.3)$ & $1.1(0.5-1.8)$ \\
\hline Aprotinin & $0.6(0.3-1.4)$ & $0.2(0.1-0.7)$ \\
\hline Tranexamic acid & $1.9(0.3-7.9)$ & $0.6(0.2-1.6)$ \\
\hline High pump volume† & $15.5(3.2-73)$ & $0.5(0.1-2.6)$ \\
\hline \multicolumn{3}{|l|}{ Surgeons } \\
\hline \multicolumn{3}{|l|}{ Group 1} \\
\hline Surgeon 1 & 1.0 (reference) & 1.0 (reference) \\
\hline Surgeon 2 & $0.7(0.2-1.9)$ & $6.9(1.6-30)$ \\
\hline Surgeon 3 & $0.5(0.2-1.5)$ & $5.3(1.3-21)$ \\
\hline Surgeon 4 & $0.5(0.2-1.4)$ & $8.7(2.1-36)$ \\
\hline \multicolumn{3}{|l|}{ Group 2} \\
\hline Surgeon 5 & 1.0 (reference) & 1.0 (reference) \\
\hline Surgeon 6 & $2.0(0.8-5.4)$ & $3.3(1.5-7.4)$ \\
\hline Surgeon 7 & $1.4(0.5-3.7)$ & $4.4(2.0-9.7)$ \\
\hline Surgeon 8 & $2.2(0.7-6.4)$ & $2.8(1.2-6.8)$ \\
\hline
\end{tabular}

$H C T$, Hematocrit; $C l$, confidence interval. *Bold values indicate significant associations, $P<.05$. $†$ High pump volume: 750 pentastarch of total of 2000 priming volume. \$Surgeons compared only with other surgeons using similar institutional practices of initial volume of fluid and colloid added to extracorporeal circulation and antifibrinolytic agent: group 1, $750 \mathrm{~mL}$ pentastarch of a total of $2000 \mathrm{~mL}$ of priming volume; group 2, $500 \mathrm{~mL}$ of pentastarch of total of $1400 \mathrm{~mL}$ of priming solution.

Eighteen $(25 \%)$ of 68 patients whose procedures were performed off pump, a similar proportion to the population with operations performed on pump, had excessive postoperative hemorrhage (data not shown).

In multivariate analysis (Table 4) of patients whose procedures were performed on pump, factors associated with intraoperative anemia were those that might reflect greater hemodilution, such as female sex, smaller body surface area, lower preoperative hemoglobin level, and larger initial total and colloid volume in the bypass circuit.

None of these factors were associated with postoperative bleeding. In contrast, excessive postoperative hemorrhage was associated with male sex and was less likely in patients who received aprotinin. After adjusting for these factors, certain surgeons had significantly higher rates of postoperative bleeding. When multivariate linear analyses were performed evaluating blood loss and lowest intraoperative anemia as continuous variables, the same factors were significantly associated as were associated with the excessive postoperative hemorrhage and severe intraoperative anemia as defined dichotomously.

Severe intraoperative anemia was a stronger predictor of packed cell transfusions, whereas excessive postoperative hemorrhage was a stronger predictor of receiving coagula- tion factors. Of the patients who had severe intraoperative anemia, $88 \%$ were transfused packed cells perioperatively compared with $72 \%$ who had excessive postoperative hemorrhage, whereas of the patients who met the criteria for excessive postoperative hemorrhage, 50\% received fresh frozen plasma and $33 \%$ received platelets compared with only $27 \%$ receiving fresh frozen plasma and $17 \%$ receiving platelets among the patients with intraoperative anemia (Table 5).

\section{Discussion}

We have shown that intraoperative anemia and excessive bleeding were associated with different risk factors and appear to be independent phenomena. Both were common and associated with worse outcomes and increased blood product transfusion. Some of the risk factors found are potentially modifiable, which suggests that efforts could be undertaken to decrease the frequency of these 2 entities and thereby possibly improve outcomes in patients undergoing CABG.

One important inference from these results is that perioperative blood product requirements in cardiac surgery might not all be due to excessive bleeding. Patients who had intraoperative anemia had the same mean volume of mediastinal drainage postoperatively as patients who did not 
TABLE 5. Comparison of different blood product use in patients undergoing CABG on pump with intraoperative hematocrit levels of less than $19 \mathrm{~g} / \mathrm{L}$ or mediastinal drainage of greater than $1 \mathrm{~L}$

\begin{tabular}{|c|c|c|c|}
\hline Periop blood products & $\begin{array}{c}\text { Lowest intraop HCT }<19 \% \\
\text { vs } \geq 19 \%, \text { RR }(95 \% \text { CI) }\end{array}$ & $\begin{array}{c}\text { Mediastinal drainage }>1 \mathrm{~L} \\
\text { vs }<1 \mathrm{~L} \text { in the first } 12 \mathrm{~h} \text {, } \\
\mathrm{RR}(95 \% \mathrm{Cl})\end{array}$ & $\begin{array}{c}\text { Anemia vs bleeding, } \\
\text { RR }(95 \% \mathrm{CI})\end{array}$ \\
\hline No. of patients & 131 & 140 & \\
\hline Packed cells & $7.2(4.3-12)$ & $3.0(2.1-4.4)$ & $2.8(1.5-5.3)$ \\
\hline Fresh frozen plasma & $1.6(1.2-2.2)$ & $4.4(3.5-5.7)$ & $0.6(0.4-0.9)$ \\
\hline Platelets & $1.4(0.9-2.0)$ & $3.3(2.6-4.3)$ & $0.6(0.4-0.8)$ \\
\hline
\end{tabular}

$H C T$, Hematocrit; $R R$, risk ratio; $\mathrm{Cl}$, confidence interval.

have intraoperative anemia, and less than one third had excessive postoperative bleeding. Many patients who had intraoperative anemia in this study received a transfusion both in the operating room and postoperatively. ${ }^{21,22}$ The differences in the use of coagulation factors associated with mediastinal drainage compared with severe intraoperative anemia also support the hypothesis that clinicians are responding differently to the 2 transfusion triggers.

In this study the strong association of intraoperative anemia with female sex, lower preoperative hemoglobin level, smaller body surface area, and larger total and colloid volume in the initial pump priming solution suggests that hemodilution was an important contributor to this problem. This is also supported by the almost negligible risk (4\%) in patients undergoing off-pump procedures. The occurrence of intraoperative anemia has been shown to be an independent predictor of perioperative mortality and morbidity, ${ }^{4,6}$ and strategies to decrease its occurrence might improve outcomes in this population. These strategies would include first decreasing preoperative anemia with practices such as limiting preoperative $\mathrm{e}^{23}$ blood taking, use of more microlaboratory techniques, and iron supplementation and perhaps erythropoietin in patients awaiting elective operations. Second, because of the strong association with the larger volumes of priming solution with higher volumes of colloid in the extracorporeal circuit, the volume should be adjusted, taking into consideration patient size, sex, and preoperative hemoglobin concentration, rather than using a fixed volume.

This is supported by previous reports ${ }^{24,25}$ of decreases in intraoperative packed cell use with the use of lower prime volume. In a recent study, ${ }^{26}$ use of hetastarch increased the likelihood of patients undergoing coronary artery surgery receiving 3 or more units of any blood product. The hetastarch might have contributed to greater hemodilution and lower intraoperative hematocrit levels, as well as increased bleeding. ${ }^{26}$ In our study, higher pump volumes and use of more colloid was strongly associated with intraoperative anemia but not with excessive postoperative bleeding. In the study hospitals, the medium-molecular-weight starches (Pentaspan; Bristol Myers Squibb Canada), for which there are less data on increased bleeding risk, are used. ${ }^{27}$ The doses of hetastarch used during bypass were fixed and in most patients were less than $15 \mathrm{~mL} / \mathrm{kg}$.

Although excessive postoperative hemorrhage has been reported to be one of the most common complications of cardiac surgery, ${ }^{12}$ relatively few studies have examined this outcome ${ }^{11,28}$ because most studies examined perioperative transfusions. It has been suggested that outcomes related to excessive drainage should be examined, such as resternotomy $^{29}$ and blood product transfusions; our results highlight the limitations of this approach. We noted marked differences in reoperation rates and blood product use in the 3 study sites, which were not related to rates of postoperative bleeding (Table 1) but might reflect practice differences. Therefore, analysis of factors associated with the amount of postoperative bleeding was less likely to be biased than analysis of factors associated with these management decisions. In addition, because postoperative bleeding was associated with longer ICU lengths of stay, transfusions, and a higher mortality, we believe this definition is clinically relevant. In addition, measurement of postoperative mediastinal drainage is objective, routinely performed, and reproducible.

In a prospective study of more than 10,000 patients undergoing cardiac surgery, patient characteristics were not associated with reoperation for postoperative bleeding. ${ }^{30}$ The authors suggested that reoperation for bleeding might be more related to technical surgical factors rather than patient-related factors. Two other studies have demonstrated that postoperative hemorrhage was associated with surgeon, ${ }^{11,13}$ and meticulous surgical technique is frequently mentioned ${ }^{16}$ as an important means to decrease blood product use in cardiac surgery. These findings are consistent with ours, in which male sex was the only patient characteristic and surgeon was strongly associated with this outcome. We speculated that the association with male sex might have occurred because of the assumption that female subjects have higher bleeding risks, which might have resulted in more meticulous surgical practices in female subjects.

We attempted to include in the multivariate analysis the important factors believed to be associated with increased risk of bleeding and transfusion requirements, as well as the factors that differed among the 3 hospitals. There might be 
other unmeasured factors that we did not account for that could explain some of the results. Certain variables were also very tightly correlated, such as antifibrinolytic use, volume of prime used in the extracorporeal circuit, and particular surgeon, which limit some of the inferences that can be drawn from the results. In this study neither larger total circuit volumes nor larger HES volumes were associated with excessive mediastinal drainage. Although there have been recommendations that HES not be used in patients undergoing cardiac surgery because of concerns of excess bleeding, we did not find that association in this study, even after adjusting for many clinical variables. The almost exclusive use of aprotinin by the center using the larger volumes of HES might account for our not finding an effect.

There were very few patients who had been exposed to clopidogrel, and no patient had been prescribed lowmolecular-weight heparin. We also studied only patients who had bypass procedures alone, without any valvular procedures, and therefore the conclusions might not be applicable to these patient populations. Although repeat surgical interventions were not associated with an increased risk of bleeding in this study, the study might have been underpowered to evaluate this association.

\section{Conclusions}

Our results suggest that many factors contribute to triggers to blood product transfusion in cardiac surgery. Reducing these triggers requires different strategies, including methods to decrease hemodilution, especially in small female subjects who might have anemia before surgical intervention. This could include identification and treatment of preoperative anemia and re-evaluation of the design of the bypass circuit. Adjustment of prime volumes with less HES use and use of techniques such as retrograde autologous prime might also be beneficial. On the other hand, postoperative bleeding can be reduced with the use of aprotinin. However, given the association of surgeon with this outcome in this study, meticulous surgical technique appears to be critical to decreasing excessive postoperative bleeding.

We thank Dr Felix Ma, the cardiac surgery departments, and the perfusionists in the 3 participating hospitals for their cooperation and assistance with the project. We also thank Catherine Michaud and Esther Tomkee for secretarial assistance and Eric Tremblay and Mylene Lagace for assistance with data entry.

\section{References}

1. Goodnough LT, Johnston M, Toy PT, the Transfusion Medicine Academic Award Group. The variability of transfusion practice in coronary artery bypass surgery. JAMA. 1991;265:86-90.

2. Belisle S, Hardy JF. Hemorrhage and the use of blood products after adult cardiac operations: myths and realities. Ann Thorac Surg. 1996; 62:1908-17.

3. Karkouti K, Cohen M, McCluskey SA, et al. A multivariate model for predicting the need for blood transfusion in patients undergoing first-time elective coronary bypass graft surgery. Transfusion. 2001;41:1193-203.
4. Parr KG, Patel MA, Dekker R, et al. Multivariate predictors of blood product use in cardiac surgery. J Cardiothorac Vasc Anesth. 2003;17:176-81.

5. Hardy JF, Belisle S. Natural and synthetic antifibrinolytics in adult cardiac surgery: efficacy, effectiveness and efficiency. Can J Anaesth. 1994;41:1104-12.

6. DeFoe GR, Ross CS, Olmstead EM, et al. Lowest hematocrit on bypass and adverse outcomes associated with coronary artery bypass grafting. Northern New England Cardiovascular Disease Study Group. Ann Thorac Surg. 2001;71:769-76.

7. Hardy JF, Martineau R, Couturier A, et al. Influence of haemoglobin concentration after extracorporeal circulation on mortality and morbidity in patients undergoing cardiac surgery. Br J Anaesth. 1998;81 (Suppl 1):38-45.

8. Fang WC, Helm RE, Krieger KH, et al. Impact of minimum hematocrit during cardiopulmonary bypass on mortality in patients undergoing coronary artery surgery. Circulation. 1997;96(Suppl II):III.

9. Ferraris VA, Colonel MC, Gildengorin V. Predictors of excessive blood use after coronary artery bypass grafting: a multivariate analysis. J Thorac Cardiovasc Surg. 1989;98:492-7.

10. Magovern JA, Sakert T, Benckart DH, et al. A model for predicting transfusion after coronary artery bypass grafting. Ann Thorac Surg. 1996;61:27-32.

11. Vander Salm TJ, Kaur S, Lancey RA, et al. Reduction of bleeding after heart operations through the prophylactic use of epsilon-aminocaproic acid. J Thorac Cardiovasc Surg. 1996;112:1098-107.

12. Levi M, Cromheecke ME, de Jonge E, et al. Pharmacological strategies to decrease excessive blood loss in cardiac surgery: a metaanalysis of clinically relevant endpoints. Lancet. 1999;354:1940-7.

13. Ferraris VA, Ferraris SP, Joseph O, et al. Aspirin and postoperative bleeding after coronary artery bypass grafting. Ann Surg. 2002;235:820-7.

14. Munoz JJ, Birkmeyer NJ, Dacey LJ, et al. Trends in rates of reexploration for hemorrhage after coronary artery bypass surgery. Northern New England Cardiovascular Disease Study Group. Ann Thorac Surg. 1999;68:1321-5.

15. Shapira OM, Aldea GS, Treanor PR, et al. Reduction of allogeneic blood transfusions after open heart operations by lowering cardiopulmonary bypass prime volume. Ann Thorac Surg. 1998;65:724-30.

16. Helm RE, Rosengart TK, Gomez M, et al. Comprehensive multimodality blood conservation: 100 consecutive CABG operations without transfusion. Ann Thorac Surg. 1998;65:125-36.

17. Cormack JE, Forest RJ, Groom RC, et al. Size makes a difference: use of a low-prime cardiopulmonary bypass circuit and autologous priming in small adults. Perfusion. 2000;15:129-35.

18. Parsonnet V, Dean D, Bernstein AD. A method of uniform stratification of risk for evaluating the results of surgery in acquired adult heart disease. Circulation. 1989;79(Suppl I):I3-12.

19. Kirklin JW, Barratt-Boyes BG. Cardiac surgery. New York: John Wiley; 1986.

20. Hartstein G, Janssens M. Treatment of excessive mediastinal bleeding after cardiopulmonary bypass. Ann Thorac Surg. 1996;62:1951-4.

21. A report by the American Society of Anesthesiologists Task Force on Blood Component Therapy: practice guidelines for blood component therapy. Anesthesiology. 1996;84:732-47.

22. Consensus conference. Perioperative red blood cell transfusion. JAMA. 1988;260:2700-3.

23. Karski JM, Mathieu M, Cheng D, et al. Etiology of preoperative anemia in patients undergoing scheduled cardiac surgery. Can J Anaesth. 1999;46:979-82.

24. Baufreton C, de Brux JL, Binuani P, et al. A combined approach for improving cardiopulmonary bypass in coronary artery surgery: a pilot study. Perfusion. 2002;17:407-13.

25. Fromes Y, Gaillard D, Ponzio O, et al. Reduction of the inflammatory response following coronary bypass grafting with total minimal extracorporeal circulation. Eur J Cardiothorac Surg. 2002;22:527-33.

26. Avorn J, Patel M, Levin R, Winkelmayer WC. Hetastarch and bleeding complications after coronary artery surgery. Chest. 2003;124:1437-42.

27. Wilkes MM, Navickis RJ, Sibbald WJ. Albumin versus hydroxyethyl starch in cardiopulmonary bypass surgery: a meta-analysis of postoperative bleeding. Ann Thorac Surg. 2001;72:527-33. 
28. Despotis GJ, Filos KS, Zoys TN, et al. Factors associated with excessive postoperative blood loss and hemostatic transfusion requirements: a multivariate analysis in cardiac surgical patients. Anesth Analg. 1996;82:13-21.

29. Hammermeister KE, Burchfiel C, Johnson R, Grover FL. Identification of patients at greatest risk for developing major complications at cardiac surgery. Circulation. 1990;82(Suppl IV):IV380-9.
30. Munoz JJ, Birkmeyer NJ, Birkmeyer JD, et al. Is epsilon-aminocaproic acid as effective as aprotinin in reducing bleeding with cardiac surgery?: a meta-analysis. Circulation. 1999;99:81-9. 


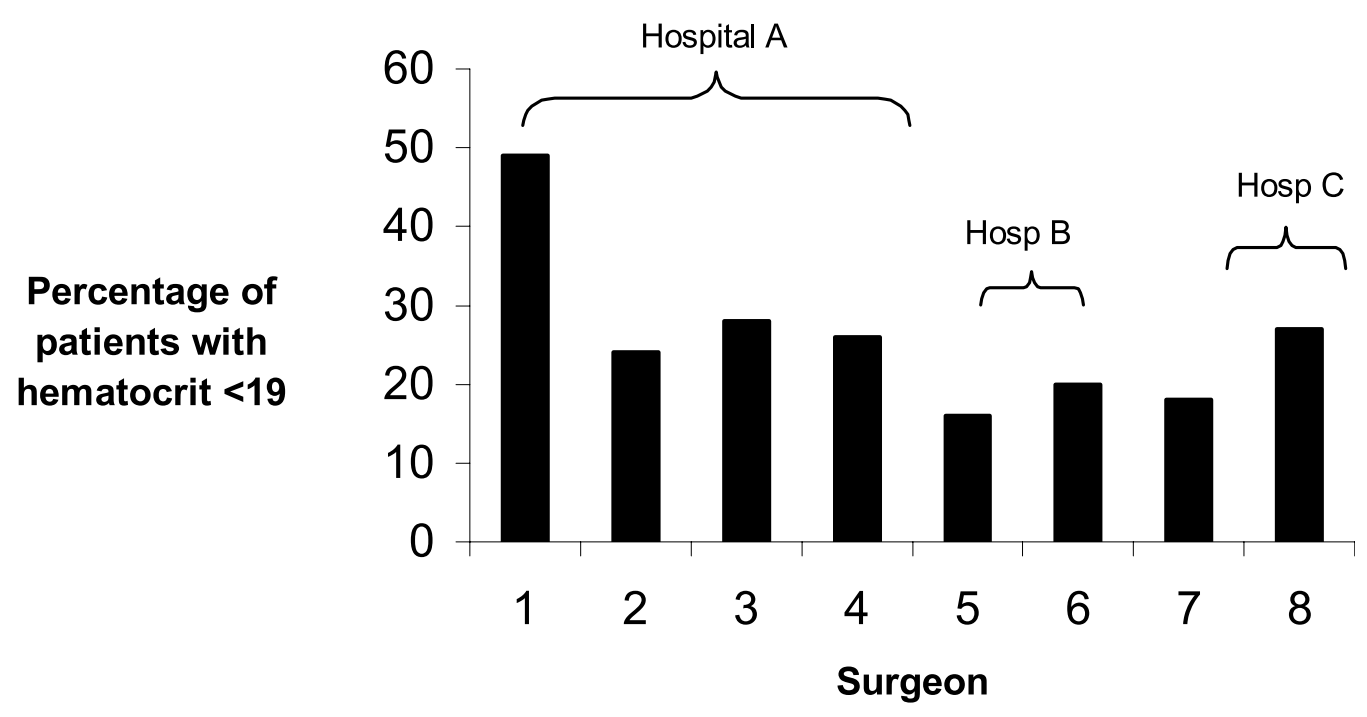

Figure E1. Proportion of patients with intraoperative anemia by hospital and surgeon.

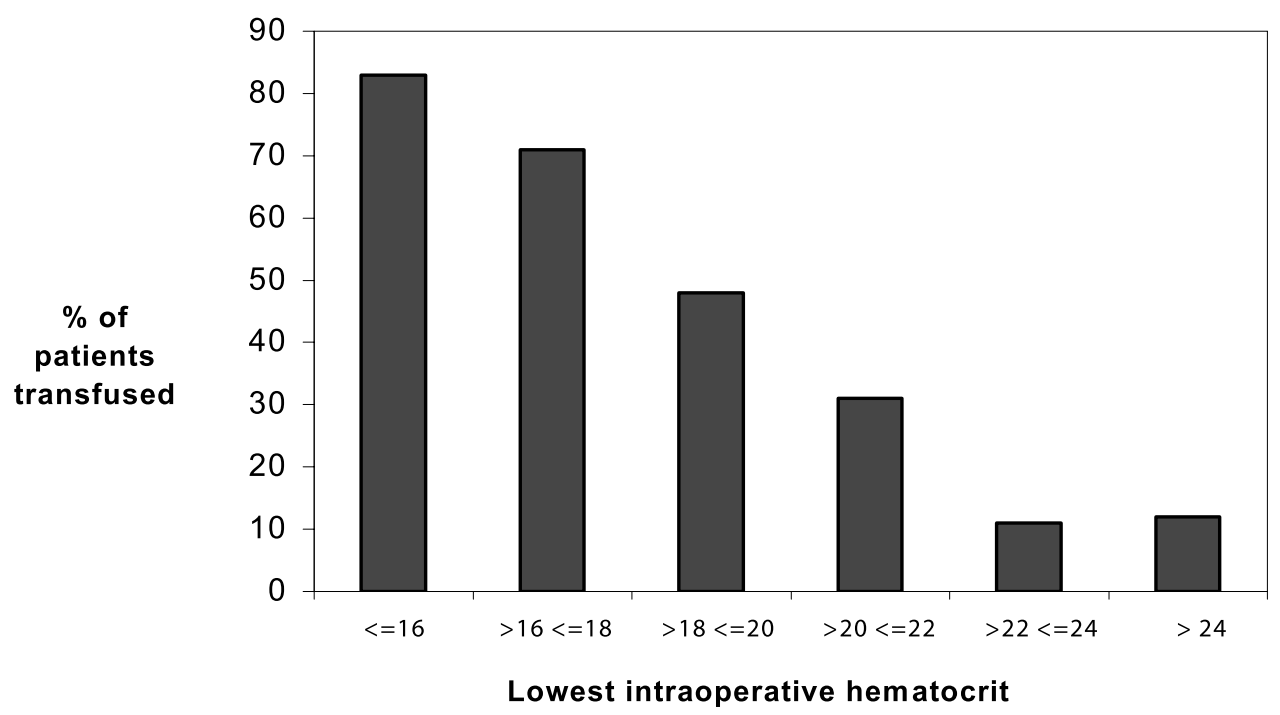

Figure E2. Proportion of patients transfused on the basis of lowest recorded hematocrit level. 


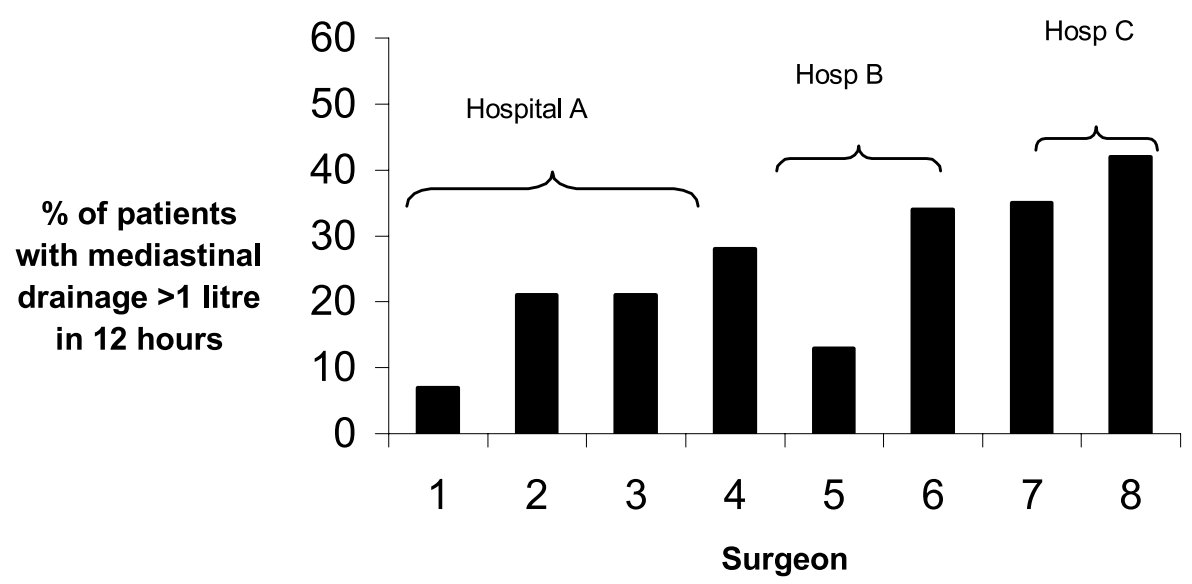

Figure E3. Comparison of proportion of patients who had mediastinal drainage of greater than $1 \mathrm{~L}$ in the first 12 hours by hospital and surgeon.

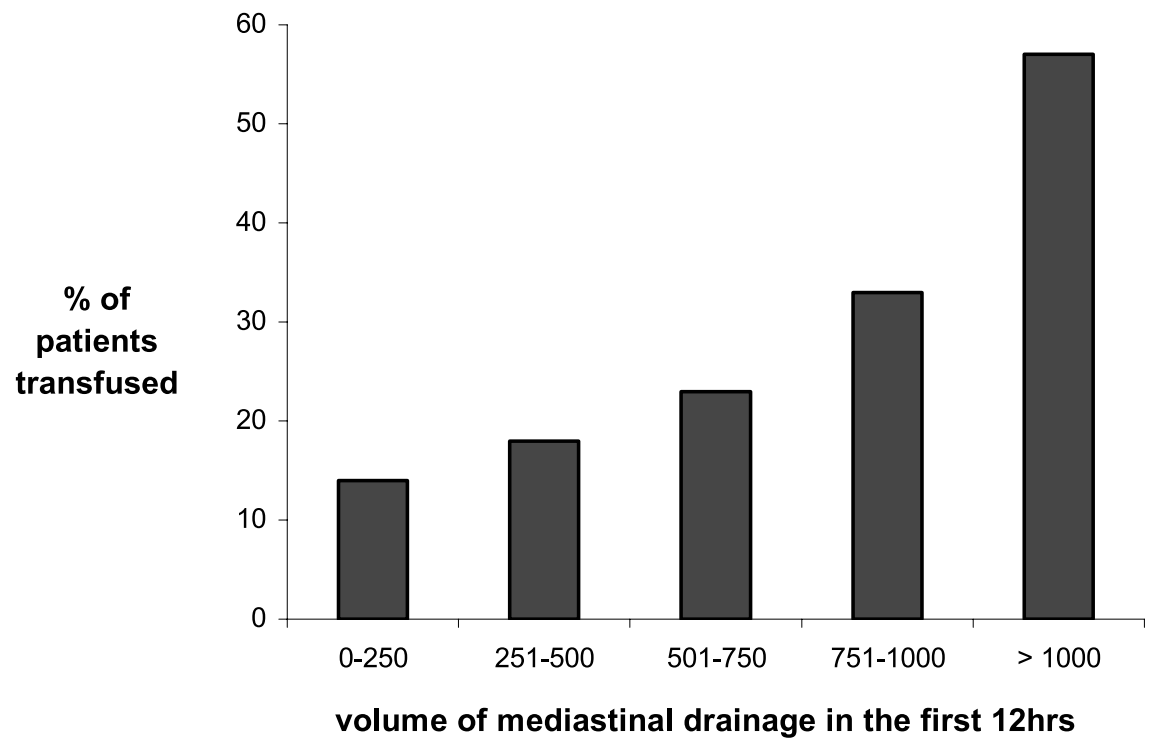

Figure E4. Proportion of patients transfused on the basis of volume of mediastinal drainage in the first 12 hours postoperatively. 